\title{
Stacking Characteristics of Composite Cardboard Boxes
}

\author{
R. B. Keller AND B. A. Hiemstra \\ Department of Mechanical Engineering and Applied Mechanics \\ The University of Michigan \\ Ann Arbor, Michigan 48109
}

(Received June 20, 1983)

\begin{abstract}
This paper presents a simplified model and method for finding the deflection characteristics of stacked cardboard boxes, provided the load-deflection characteristic of the box is known. A computer program, based on this model, allows the stability of stacked boxes to be investigated and to indicate the limits to the height of the stack and box parameters required to prevent stack toppling.
\end{abstract}

\section{INTRODUCTION}

CORREGATED CARDBOARD BOXES OR CARTONS ARE USED AS SHIPPING containers and protective covers for a wide variety of products. In order to provide sufficient strength for stacking, the boxes often consist of a composite structure, as shown in Figure 1. Four vertical wood corner posts, glued to cardboard strips, are inserted at the corners of the carton. These L-shaped wood strips take the major portion of the stacking load and are stabilized by the cardboard strips and the carton walls [1].

The stacking characteristics of the boxes are important because storage space is often best utilized if the boxes are stacked one atop the other. The vertical deflection of the cartons is determined by the weight and stiffness of the cartons; the lateral deflection is determined by these same factors plus the location of the center of gravity of the carton and its contents. If the center of gravity is not in the geometric center of the box, then the stack has a tendency to "lean" or deflect laterally. Then as the height of the stack is increased the lateral deflection increases until the center of gravity of the top-most carton falls outside the base of the bottom carton and stack becomes unstable and topples.

The stiffness of the carton, or its load-deflection characteristic, is a function of the carton design and fabrication techniques. Also moisture and aging tend to reduce the load-deflection characteristics of the carton. 


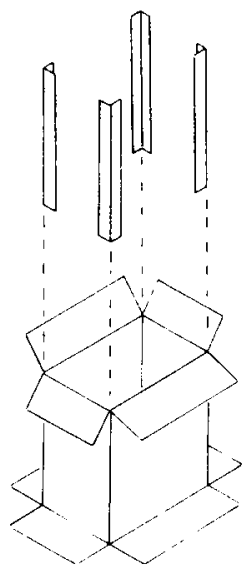

Figure 1. Typical composite carton.

\begin{abstract}
ANALYSIS
The corregated cardboard box, with corner posts, when sealed and containing the product, represents a complex, composite structure. Little data is available concerning the interactions among the various elements of this composite structure. In order to arrive at engineering answers, simplifying assumptions must be made. First, the entire load is carred by the four identical corner posts. The load-deflection characteristic, which could be found experimentally by loading the box between two parallel flat plates, is divided equally among the four corner posts. Further, the bottom and top surfaces of the box always remain plane and the corner posts are always normal to the bottom surface.

Consider the first or bottom box placed on the "floor"-a perfectly flat, horizontal plane; no deflections of either the upper or lower surfaces occur, since the weight of the product is carried directly into the floor via the bottom surface. The second box is placed atop the first box and the weight of the second box produces axial loading of the four corner posts of the bottom box. If the center of gravity is not in the center of the box cross section, then the posts have different loads and correspondingly different deflections; now the top surface of the bottom box is no longer parallel to the floor. Consequently the top edges of the second box are no longer directly above the bottom edges of the box, the two-box stack has deflected and is no longer vertical.

Adding a third box, atop the second, produces loads and deflections in the corner posts of the second box, as well as additional loads and deflections of the posts of the first or bottom box.

The loads and reactions on a typical box are shown in Figure 2. The loads, which are applied to the upper surface of the box, result from the reactions of
\end{abstract}




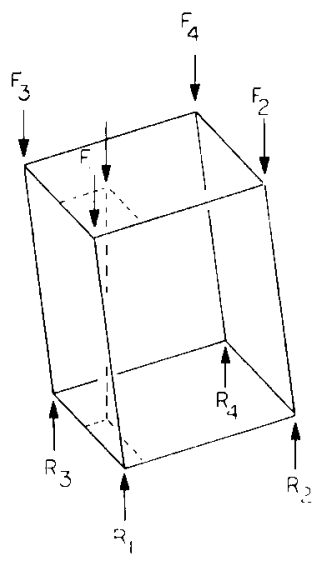

Figure 2. Isometric of box loads and reactions.

the corner posts of the box directly above and also from the weight of the box directly above. A plan view of the box is shown in Figure 3.

The reaction $R 3$ is found by taking the sum of the moments about the lower 1-2 box edge.

$$
\begin{aligned}
R_{3} & =\left[-R_{4}\left(X_{4 L}-X_{2 L}\right)+W T \cdot P X+F_{1}\left(X_{1 U}-X_{1 L}\right)+F_{2}\left(X_{2 U}-X_{2 L}\right)\right. \\
& \left.+F_{3}\left(X_{3 U}-X_{1 L}\right)+F_{4}\left(X_{4 L}-X_{2 L}\right)\right] /\left(X_{3 L}-X_{1 L}\right)
\end{aligned}
$$

The reaction $R_{2}$ is found by taking the sum of the moments about the lower 1-3 box edge

$$
\begin{aligned}
R_{2} & =\left[-R_{4}\left(Y_{4 L}-Y_{3 L}\right)+W T \cdot P Y+F_{1}\left(Y_{1 u}-Y_{1 L}\right)+F_{2}\left(Y_{2 u}-Y_{1 L}\right)\right. \\
& \left.+F_{3}\left(Y_{3 U}-Y_{3 L}\right)+F_{4}\left(Y_{4 l}-Y_{3 L}\right)\right] /\left(Y_{2 L}-Y_{1 L}\right)
\end{aligned}
$$

The reaction $R_{1}$ is found from the sum of the vertical forces,

$$
R_{1}=W T+F_{1}+F_{2}+F_{3}+F_{4}-\left(R_{2}+R_{3}+R_{4}\right)
$$

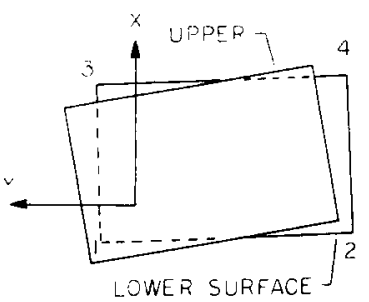

Figure 3. Plan view of upper and lower surfaces of box. 
The reaction of the fourth corner post, $R_{4}$, must be found using the following iterative process: (a) assume a value for $R_{4}$; solve for $R_{1}, R_{2}$, and $R_{3}$ using the eqns. (1), and (2) and (3). (b) Find the deflections of the three corner posts using their reactions and the load-deflection characteristic. (c) Find the equation of the plane which contains the upper points of posts 1, 2 and 3. (d) Find the upper point of post 4 which lies in this plane and (e) use the deflection between the lower and upper points of post 4 to find the reactive $R_{4}$ load from the load-deflection characteristic. Compare this calculated value of $R_{4}$ with the assumed value of $R_{4}$ and repeat the calculation until the two values converge. The equations for steps (c) and (d) are given in the appendix.

\section{Computer Program}

The analysis is readily implemented on the digital computer. Essentially the program consists of two nested iterative loops. The inner post loop is called the "box loop" and represents the solutions for the loads and deflections for a single box within the stack. The outer loop is the "stack loop" which calls the box loop repeatedly until the loads and deflections of each box in the stack are evaluated. The convergence criterion for the box loop is that the difference between the assumed and calculated values of $R_{4}$ must be less than $0.2 \%$ of the total reactive load on the posts. The convergence criterion for the stack loop is that the difference between successive calculations of the lateral deflection of the topmost box must be less than $2 \%$ of the previous deflection value.

The organization of the program can be outlined using a simple example. Suppose that a stack totaling $\mathbf{4}$ boxes is to be examined. First calculations are made for a two-box stack until convergence of the post reaction load and also the deflection of the upper edge of the second box is reached. Then another box is added, producing a three-box stack. Again the reactions of the corners of the second box and the first box are found, and the deflection of the upper edge of the third box is determined. Finally the fourth box is added to the stack, the calculations are repeated to determine the reactions on the posts of the third, second and first boxes and the deflection of the upper edge of the fourth box is found. The topple condition is reached whenever the center of gravity of the topmost box falls outside the base of the bottom box.

\section{RESULTS AND DISCUSSION}

Computer runs were made using the data sets shown in Table 1. Nominal or reference values were chosen to be representative of a typical carton; width, $20 \mathrm{in}$; depth, $20 \mathrm{in}$; height $30 \mathrm{in}$; weight $100 \mathrm{lbf}$, and with the c.g. located in a plane 15 in. above the bottom of the carton. For each data set the c.g. location in the y direction was held constant at 10 in. while the c.g. location in the $x$ direction was varied in 1 in. increments from the edge of the box. Two load-deflection characteristics for the box and posts were used, linear and nonlinear, as shown in Figure 4. The stiffness or spring constant of the linear characteristic was $3200 \mathrm{lbf} / \mathrm{in}$. For the nonlinear characteristic the load 
Table 1. Summary of Data Sets.

\begin{tabular}{rrrrrll}
\hline Set No. & WT & HT & ZW & SF & $\begin{array}{c}\text { No. of } \\
\text { boxes }\end{array}$ & \multicolumn{1}{c}{ Comment } \\
\hline 1 & 100 & 30 & 15 & 1. & 6 & nominal \\
2 & 100 & 30 & 15 & 0.5 & 6 & $0.5 \mathrm{SF}$ \\
3 & 100 & 30 & 15 & 0.2 & 6 & $0.2 \mathrm{SF}$ \\
4 & 100 & 30 & 15 & 0.1 & 6 & $0.1 \mathrm{SF}$ \\
5 & 200 & 30 & 15 & 2. & 6 & $2 \mathrm{WT}, 2 \mathrm{SF}$ \\
6 & 50 & 30 & 15 & 1. & 6 & $0.5 \mathrm{WT}$ \\
7 & 50 & 30 & 15 & 0.5 & 6 & $0.5 \mathrm{WT}, 0.5 \mathrm{SF}$ \\
8 & 100 & 15 & 15 & 1.0 & 6 & $0.5 \mathrm{HT}$ \\
9 & 100 & 15 & 15 & 0.5 & 6 & $0.5 \mathrm{HT}, 0.5 \mathrm{SF}$ \\
10 & 100 & 60 & 15 & 1.0 & 6 & $2 \mathrm{HT}$ \\
11 & 100 & 60 & 15 & 2. & 6 & $2 \mathrm{HT}, 2 \mathrm{SF}$ \\
12 & 100 & 30 & 0 & 1.0 & 6 & ZW $=0$ \\
13 & 100 & 30 & 30 & 1.0 & 6 & ZW $=30$ \\
14 & 100 & 30 & 15 & 1.0 & 7 & 7 boxes \\
15 & 100 & 30 & 15 & 1.0 & 5 & 5 boxes \\
\hline
\end{tabular}

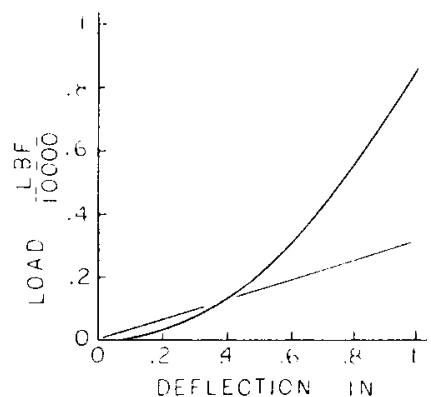

Figure 4. Load-deflection characteristics used in the data sets; linear and nonlinear.

was assumed to be proportional to the square of the deflection and was adjusted such that the lateral deflection of the upper edge of the top box was equal to that produced by the linear characteristic using the nominal data set with the c.g. 4 inches from the edge of the box.

Additional runs were made to determine the limits of allowable c.g. location before toppling occurred, using the nominal condition and c.g. heights of 0,15 , and $30 \mathrm{in}$. and using the linear force-deflection characteristic.

Results for the data sets of Table 1 are shown in Figures 5 and 6 for the linear and non-linear load-deflection characteristics, respectively. The lateral deflection of the upper edge of the topmost box is plotted as a function of the distance of the c.g. from the center of the box. In all cases, as the c.g. is moved outward from the center of the box, the deflection of the top box edge increases until topple occurs. If the height, or weight, or number of boxes is reduced, additional c.g. movement is required to produce toppling. Con- 


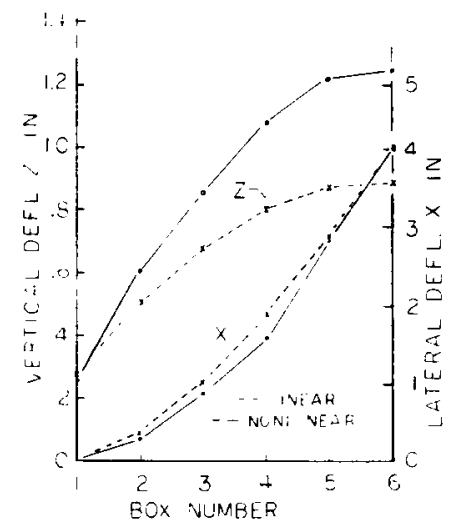

Figure 5. Lateral deflection of the top box as function of c.g. location using the linear loaddeflection characteristic. Encircled numbers correspond to the data sets of Table 1.

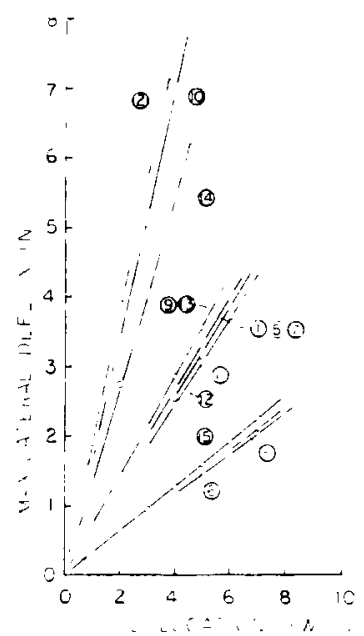

Figure 6. Lateral deflection of the top box as a function of c.g. location using the nonlinear loaddeflection characteristic. Encircled numbers correspond to the data sets of Table 1.

versely, reducing the load-deflection characteristic, or increasing the number of boxes, or the height of the boxes reduces the allowable excursion of the c.g. Doubling the weight and doubling the load-deflection characteristic or stiffness gave a c.g. movement vs. deflection curve which was nearly identical with the nominal condition. A similar result occurred when the weight and the stiffness were both halved. When the c.g. was raised to the top of the box the allowable c.g. movement was only slightly reduced; a similar small increase in stability was observed when the c.g. was lowered to the bottom of the box.

When the runs for the non-linear characteristic are examined, the general trends found for the linear case are repeated, except that for parameter excur- 


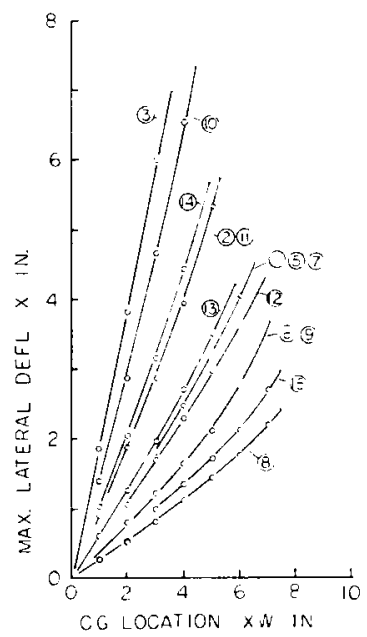

Figure 7. Lateral and vertical deflections of post 1 of the top box for the linear and nonlinear loaddeflection characteristics for data set 1.

sions which reduce the load or the force-deflection characteristic, the increase in the stability limits is not quite as large as was the case for the linear characteristic. Conversely, for those runs for which the loads were increased, or the stiffness reduced, the stability limits are slightly larger than for the linear case. These results are consistent, in that the local stiffness of the non-linear characteristic increases with increasing load or deflection and decreases with decreasing load or deflection.

Figure 7 shows the lateral and vertical deflection for each box for the two linear and non-linear force-deflection characteristics. While the lateral deflections are nearly identical (recall that the non-linear characteristic was chosen such that the lateral deflection of the top box was the same as for the linear characteristic), the vertical deflections are not. The reduced stiffness of the non-linear characteristic at reduced loads results in increased deflection at each box level.

The allowable ranges of c.g. locations for stability is shown in Figure 8 for the nominal data set for c.g. heights of 0,15 , and 30 inches. The unevenness of the contours is probably caused by a combination of the assumptions used in the model, along with the convergence errors.

\section{SYMBOLS}

F force applied to top of post

HT height of box

PX distance of weight of upper box from 1-3 edge of bottom surface of lower box

PY distance of weight of upper box from 1-2 edge of bottom surface of lower box 


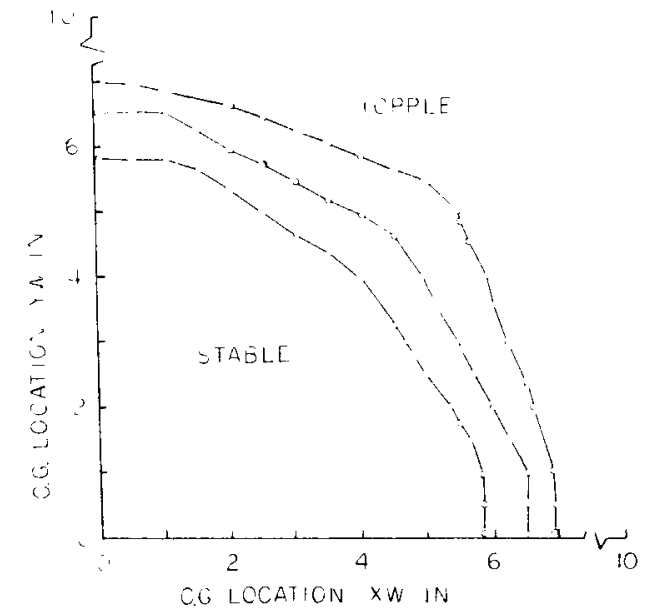

Figure 8. Allowable latera/ c.g. locations for c.g. at bottom, middle, and top of box. (Data sets 12,1 , and 13 , respective/yl

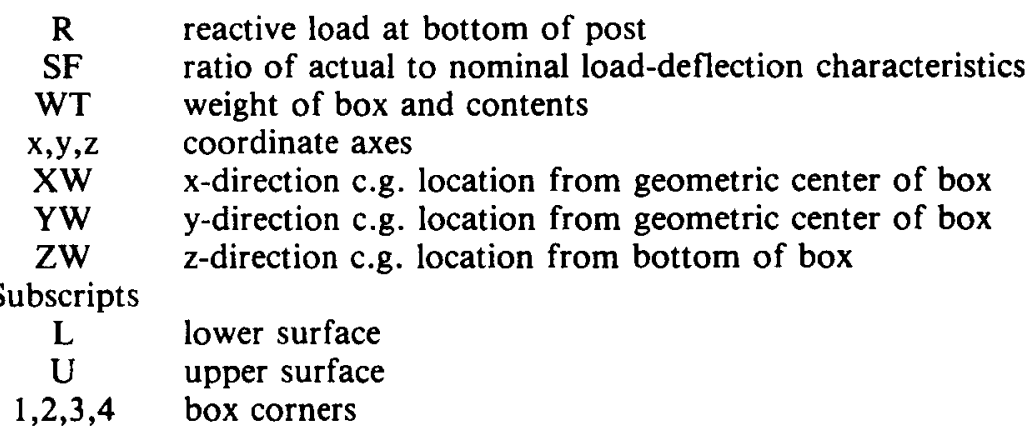

\section{CONCLUSION}

A model for determining the deflection and stability characteristics of stacked boxes has been developed and programmed on the digital computer. This program was used to investigate the deflection and stability characteristics for a typical composite box using both linear and nonlinear deflection characteristics. Results and trends appear reasonable and indicate that the model is valid and useful.

\section{ACKNOWLEDGEMENT}

The support of the Whirlpool Corporation in this work is appreciated.

\section{REFERENCES}

1. Bodig, J. and Jayne, B. A. "Mechanics of Wood and Wood Composites" Van NostrandReinhold, N.Y. 1982.

2. Rektorys, K., "Survey of Applicable Mathematics" M.I.T. Press, Cambridge, Mass, 1969. 


\section{APPENDIX}

1. Find a point $x_{1}, y_{1}, z_{1}$ which is a distance $\mathrm{d}$ along a normal from the point $x_{0}, y_{0}, z_{0}$. The line is normal to the plane $A x+B y+C z+D=0$ which contains the point $x_{0}, y_{0}, z_{0}$.

The distance of a point above a plane is [2]

$$
d=\frac{A x_{1}+B y_{1}+C z_{1}+D}{\sqrt{A^{2}+B^{2}+C^{2}}}
$$

Using the equation of a line through these two points and along the normal to the plane

$$
\frac{x_{1}-x_{0}}{A}=\frac{y_{1}-y_{0}}{B}=\frac{z_{1}-z_{0}}{C}
$$

yields

$$
\begin{aligned}
& z_{1}=\frac{\left[d \sqrt{A^{2}+B^{2}+C^{2}}-D+B\left(B / C z_{0}-y_{0}\right)+A\left(A / C z_{0}-x_{0}\right)\right]}{C\left[\frac{A^{2}}{C^{2}}+\frac{B^{2}}{C^{2}}+1\right]} \\
& y_{1}=B / C\left[z_{1}-z_{0}\right]+y_{0} \\
& x_{1}=A / D\left[z_{1}-z_{0}\right]+x_{0}
\end{aligned}
$$

2. Find the equation of a plane containing 3 non-collinear points. In matrix form, the equation is

$$
\left[\begin{array}{llll}
x & y & z & 1 \\
x_{1} & y_{1} & z_{1} & 1 \\
x_{2} & y_{2} & z_{2} & 1 \\
x_{3} & y_{3} & z_{3} & I
\end{array}\right]=0
$$

Expanding and collecting terms gives

$$
A x+B y+C z+D=0
$$

where

$$
\begin{aligned}
& A=z_{1}\left(y_{3}-y_{2}\right)+z_{2}\left(y_{1}-y_{3}\right)+z_{3}\left(y_{2}-y_{1}\right) \\
& B=z_{1}\left(x_{2}-x_{3}\right)+z_{2}\left(x_{3}-x_{1}\right)+z_{3}\left(x_{1}-x_{2}\right) \\
& C=y_{1}\left(x_{3}-x_{2}\right)+y_{2}\left(x_{1}-x_{3}\right)+y_{3}\left(x_{2}-x_{1}\right) \\
& D=x_{1}\left(y_{3} z_{3}-y_{2} z_{2}\right)+x_{2}\left(y_{1} z_{3}-y_{3} z_{1}\right)+x_{3}\left(y_{2} z_{1}-y_{1} z_{2}\right)
\end{aligned}
$$


3. Locate the fourth vertex of a parallelogram in a plane which is determined by the vertices 1,2 and 3 .

Writing the equation for point $4^{\prime}$ which is on the line which connects points 2 and 3

$$
\frac{x_{4}^{\prime}-x_{2}}{x_{3}-x_{2}}=\frac{y_{4}^{\prime}-y_{2}}{y_{3}-y_{2}}=\frac{z_{4}^{\prime}-z_{2}}{z_{3}-z_{2}}
$$

Since point $4^{\prime}$ is equidistance between points 2 and 3 , then

$$
\begin{aligned}
& x_{4}{ }^{\prime}=\left(x_{2}+x_{3}\right) / 2 \\
& y_{4}{ }^{\prime}=\left(y_{2}+y_{3}\right) / 2 \\
& z_{4}{ }^{\prime}=\left(z_{2}+z_{3}\right) / 2
\end{aligned}
$$

Also since point $4^{\prime}$ is equidistant between point 1 and 4 , point 4 is written as

$$
\begin{aligned}
& x_{4}=x_{2}+x_{3}-x_{1} \\
& y_{4}=y_{2}+y_{3}-y_{2} \\
& z_{4}=z_{2}+z_{3}-z_{1}
\end{aligned}
$$

